\title{
TOWARDS LARGE-SCALE DATA ANNOTATION OF AUDIO FROM WEARABLES: VALIDATING ZOONIVERSE ANNOTATIONS OF INFANT VOCALIZATION TYPES
}

\author{
Chiara Semenzin ${ }^{1}$, Lisa Hamrick ${ }^{2}$, Amanda Seidl $^{2}$, Bridgette Kelleher ${ }^{2}$, Alejandrina Cristia ${ }^{1 *}$ \\ ${ }^{1}$ Laboratoire de Sciences Cognitives et de Psycholinguistique, Département d'études cognitives, \\ ENS, EHESS, CNRS, PSL University, Paris, France \\ ${ }^{2}$ SLHS, Purdue University, West Lafayette, IN, USA
}

\begin{abstract}
Recent developments allow the collection of audio data from lightweight wearable devices, potentially enabling us to study language use from everyday life samples. However, extracting useful information from these data is currently impossible with automatized routines, and overly expensive with trained human annotators. We explore a strategy fit to the 21 st century, relying on the collaboration of citizen scientists. A large dataset of infant speech was uploaded on a citizen science platform. The same data were annotated in the laboratory by highly trained annotators. We investigate whether crowdsourced annotations are qualitatively and quantitatively comparable to those produced by expert annotators in a dataset of children at high- and low-risk for language disorders. Our results reveal that classification of individual vocalizations on Zooniverse was overall moderately accurate compared to the laboratory gold standard. The analysis of descriptors defined at the level of individual children found strong correlations between descriptors derived from Zooniverse versus laboratory annotations.
\end{abstract}

Index Terms - Speech corpora, wearable, data annotation, crowd-sourcing, language acquisition, infancy

\section{INTRODUCTION}

The collection of audio data has played a central role in a variety of scientific fields, allowing accurate predictions of human emotions and even diseases. Recent years have seen the rise of a novel approach to the collection speech data, thanks to astounding advancements in the field of wearable technology. From smartwatches [1] to small recorders worn

*AC acknowledges Agence Nationale de la Recherche (ANR-17CE28-0007 LangAge, ANR-16-DATA-0004 ACLEW, ANR-14-CE30-0003 MechELex, ANR-17-EURE-0017); and the J. S. McDonnell Foundation Understanding Human Cognition Scholar Award. LH acknowledges the National Institute of Deafness and Other Communication Disorders (F31DC018219). BK acknowledges the National Institute of Mental Health (K23MH111955) and the Kinley Trust. This publication uses data generated via the Zooniverse.org platform, development of which is funded by generous support, including a Global Impact Award from Google, and by a grant from the Alfred P. Sloan Foundation. by young children (e.g., LENA ${ }^{\mathrm{TM}}$ recorders; [2]), wearables allow us to capture what people say and hear as they go about a normal day. They have the potential to overcome the limitations of artificial, controlled environments, and offer tremendous promise for applications, such as in the context of mental and physical health monitoring. Nevertheless, the ease of data collection stands in contrast with the complexity of the annotation process required after data is gathered.

First, automatizing the detection of "who spoke when", a task known as diarization, has proven extremely challenging. Diarization systems for recordings are often limited in their accuracy when applied to the highly spontaneous, overlapping speech in a range of acoustic contexts found in data from wearables. Such systems are typically not resilient to variation in recording equipment, ambient noise, and speakers' idiosyncrasies, leading to error rates hovering around $70 \%$ [3]. The same challenges make any downstream task extremely hard, including even simple acoustic classification tasks. For instance, in a recent challenge, state-of-the-art systems achieved a best accuracy of around 59\% on a test set when classifying infant vocalization into 5 classes (crying, laughing, canonical, non-canonical, and junk, introduced below) [4].

In view of this, researchers collecting data from wearables therefore have had to rely on human annotation, which is slow, laborious, and may require significant training [5], but may be significantly better than automated measures (e.g.,[6]) As a result, often only a tiny percentage of the data collected is annotated (e.g., [7]): daylong infant-centered recordings require about an hour of human work to annotate 2 minutes of audio. As typical recordings are between 12 and 16 hours long, coding a single recording could take anywhere from 360 to 480 hours of intense, highly-trained labor.

One option to side-step this challenge may involve crowdsourcing. The low cost of crowd-sourcing has already facilitated data collection and analysis in a number of fields, and has become a hot topic particularly in data-driven sciences. With the surge of interest in online data collection, Mechanical Turk (MTurk) has emerged as the leading service: MTurk is an online labor market created by Amazon to assist "re- 
questers" in hiring and paying "workers" for the completion of computerized tasks. Social scientists have discovered the potential of the MTurk workforce as a large pool of participants, constantly available to complete research studies at a low cost. Today, it is not uncommon to read empirical articles that are entirely based on data collected using Mturk, and several studies have assessed its reliability [8][9], with some question marks being raised as some "workers" turn out to be bots, or poorly motivated (and potentially exploited).

Recent years have seen the rise of a fascinating crowdsourcing alternative, the one that we turn to: citizen science, a research technique that engages the public in the collection of scientific information [10]. Citizen scientists do not receive compensation and their participation to projects is voluntary and entirely motivated by the desire to contribute to research advancements and/or the pleasure they derive from the task itself. One of the most successful platforms hosting citizen science projects is Zooniverse (zooniverse.org). The website projects have allowed the public to take part in cutting-edge scientific research in a multitude of fields, from marine biology to papyrology. Zooniverse has proven extremely useful to scientists as the complexity of much of the data collected is too high to be automatically interpreted using computer algorithms [11]. At the same time, the tasks that human volunteers are asked to complete are sufficiently simple that citizens can carry them out without a background in science or any extensive training [12][13].

Citizen science may be particularly helpful when analyzing infants' data from wearables. Although this technique is increasingly common in the fields of language acquisition and health, recent challenges suggest that it remains difficult to annotate these audios automatically [3]. In response to this, Cychosz and colleagues [14] gathered more than 13k clips extracted from child vocalizations in a diverse set of crosslinguistic corpora. Annotations were obtained using the citizen science iHearUPlay platform, which successfully replicated previous results on the relationship between distributions of infant vocalization types and age. Although these results are promising, there are several limitations of their approach. First, the iHearUPlay platform relies on human interaction for data upload and annotation extraction, and the authors of that work ended up recruiting their own students and staff to complete the tasks. This is in contrast to the capabilities of Zooniverse, which has over 1.6 million users and a completely automatized API system. Additionally, recruiting students may mean that they are more experienced with the data, and thus their performance overestimates the quality of the data that would ensue from a regular citizen science crowd-sourcing effort. Second, that paper did not report on benchmark data that had been annotated in the laboratory, and thus we do not know how citizen scientists' annotations fare compared to trained annotators.

\subsection{The present work}

The current work aims at answering the question of whether annotations obtained through a citizen science platform are qualitatively and quantitatively comparable to the ones obtained from expert annotators. We will do so by examining the extent to which such classifications agree, by i) studying the correspondence between expert human annotations and Zooniverse annotations and ii) quantifying the alignment between descriptors at the child level across the two annotation settings.

Specifically, the classifications under study here are related to the complexity of early linguistic productions. Infants' early vocal production changes remarkably over the first year of life. Although infants produce speech-like productions shortly after birth, we observe both an increase in quantity and a change in quality with development [15]. In terms of quantity, we observe increases in the proportion of vocal productions that are speech-like rather than purely emotional (e.g., crying) - we will call this the linguistic ratio. As for quality, a critical milestone involves the increasingly common production of canonicalsyllables, consonant-vowel sequences that resemble those found in adult speech [16]. These may be compared to more primitive sounds, such as squeals or isolated consonants or vowels, in that their structure shows a higher complexity given by the smooth articulatory transition between a consonant and a vowel (or vice versa). In typically-developing infants, the ratio between canonical sequences and non-canonical ones - the canonical ratio - increases with age, and the development of these syllables is predictive of later language development well past the second year of life [?]. In the data we analyze below we also include children diagnosed with Angelman Syndrome, among whom canonical babbling is affected, such that canonical ratio decreases with age.

\section{METHODS}

This study was approved by the local institutional review board under the project name Neurodevelopmental Natural History Study, Purdue University, IRB-1811021381.

\subsection{Participants}

The full data set includes data from 10 children ( 6 males, 4 females; age range 11-53 months, mean=41.5 months) diagnosed with Angelman syndrome, a genetic syndrome characterized by severe language impairments, and 10 low-risk control children (6 males, 4 females; age range 4-18 months, mean=11.7 months). However, we only have data for 10 children so far. 


\subsection{Data collection}

Recordings were obtained with the Language ENvironment Analysis $\left(\mathrm{LENA}^{\mathrm{TM}}\right)$ Digital Language Processor [2], a small recorder children wore inside a breast-pocket of purposemade clothing. In our data, nearly every child contributed one daylong recording (duration 11.58-16.00 hours, mean 15.05 hours; one child contributed two recordings).

\subsection{Data pre-processing}

Segments of the daylong recording containing the key child speech were automatically extracted using LENA ${ }^{\mathrm{TM}}$ proprietary software. Specifically, the LENA ${ }^{\mathrm{TM}}$ software attempts to automatically identify whether the speaker is the target child, another child, an adult male or an adult female, with a classification accuracy of $76 \%$ [17]. A subset of utterance audio clips identified as the key child were selected for human annotation. This subset included utterance audio clips from periods of high child volubility as well as randomly selected periods throughout the day, which should contribute to generalizability of these data. Annotation was thus carried out at the segment level during lab coding.

Even short extracts of spontaneous human conversations pose serious risk of a privacy breach. Fortunately, [7] found in a laboratory experiment that human annotators can code our categories of interest (crying, laughing, canonical, etc.) when hearing the same segments divided into shorter chunks (400$600 \mathrm{~ms}$ ), obtaining a classification quality comparable to the one carried out on full segments. By using very short $(500 \mathrm{~ms})$ clips we prevent leakage of personal information: $500 \mathrm{~ms}$ clips are unlikely to contain more than two syllables, and are thus too short to reveal identifying information like addresses or names.

Therefore, segments were divided up into chunks of exactly 500ms (taking neighboring silence if necessary), before being uploaded on Zooniverse. A mapping between chunks and segments was created in the process in order to reconstruct the segment at later stages of the analysis.

\subsection{Human Expert Classification}

Coders were 20 undergraduate students working in SLHS or clinical psychology laboratories. Before annotation, coders complete an ethical conduct of research course and receive HIPAA compliance training. After this, they complete a brief training on vocal maturity that involves reviewing relevant literature and examples of various annotation categories and completing a brief quiz to test their annotation accuracy. Coders then proceed to the classification, where they make decisions on whether an utterance is a cry, laugh, noncanonical syllable, canonical syllable, or a word. Coders are also given a "Don't Code" option, which they are instructed to use if the utterance does not sound like an utterance made by the target child, or if there is significant overlapping speech or other noise in the background. Reliability is built into the annotation routine such that each utterance is coded by 3 separate coders, and only utterances where 2 of 3 coders agree are used in analyses.

\subsection{Zooniverse Classification}

Clips were uploaded on the citizen-science platform Zooniverse, and classified by annotators as belonging to one of five possible categories: (1) canonical utterance, (2) noncanonical utterance, (3) crying, (4) laughing, and (5) junk (overlapping speech, non-infant speech, silence, external sounds). The latter corresponds to the "Don't mark" category of the human expert annotation routine. Before starting the annotation, participants could walk through a tutorial explaining the classification workflow (i.e., how to interact with the Zooniverse interface), as well as how to distinguish between categories, by listening to several audio examples for each category. These examples and further explanations were also available throughout the classification workflow in a Field Guide, placed on the right side of the screen. The Field Guide could be consulted without interrupting the classification flow. A total of 3,424 individual Zooniverse users provided labels in for the larger project of which the present data set is one part.

\section{RESULTS}

There have been 169,628 labels provided for 33,880 500-ms chunks. After removing 6736 chunks that did not have at least 3 labels in agreement, we ended up with 135,720 labels for 27,144 chunks.

We further combined these chunks to reconstruct segmentlevel judgments following these rules: If all judgements were junk, then the segment was labelled as junk; then, following a hierarchy (Canonical $>$ Non - canonical $>$ Laughing $>$ Crying), segments with at least one instance of each judgement were labelled as such: e.g. if one canonical judgement was present, then the segment is labelled as canonical, otherwise if a non-canonical judgement was present, the segment is labelled as non-canonical, and so on. This led to 11,593 segments for which we had both majority coding for Zooniverse and our gold standards. Since the segments average 1.12 seconds in length, this represents roughly 3.8 hours of data. Please see Figures 1-2 for the frequency of each of the labels.

\subsection{Correspondence between lab \& Zooniverse annota- tion at the level of segments}

In this section, we look at to what extent Zooniverse and lab annotations match at the level of individual segments. Each data point is one segment (one "vocalization"). The quality of results is assessed using a confusion matrix, and visualized 


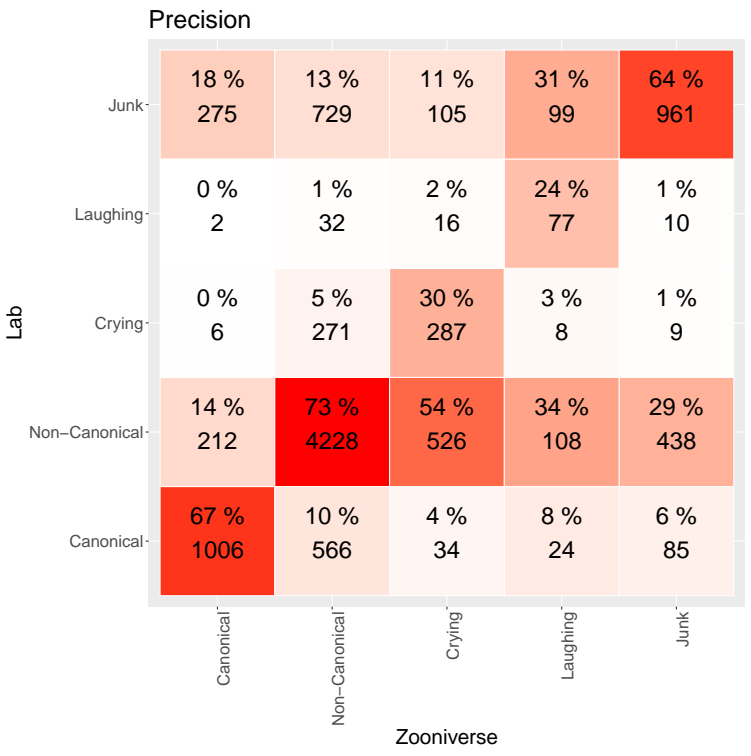

Fig. 1. Precision (i.e., category $X$ labels in Zooniverse that were classified as $\mathrm{Y}$ labels by lab annotators) in raw number of segments and percentage.

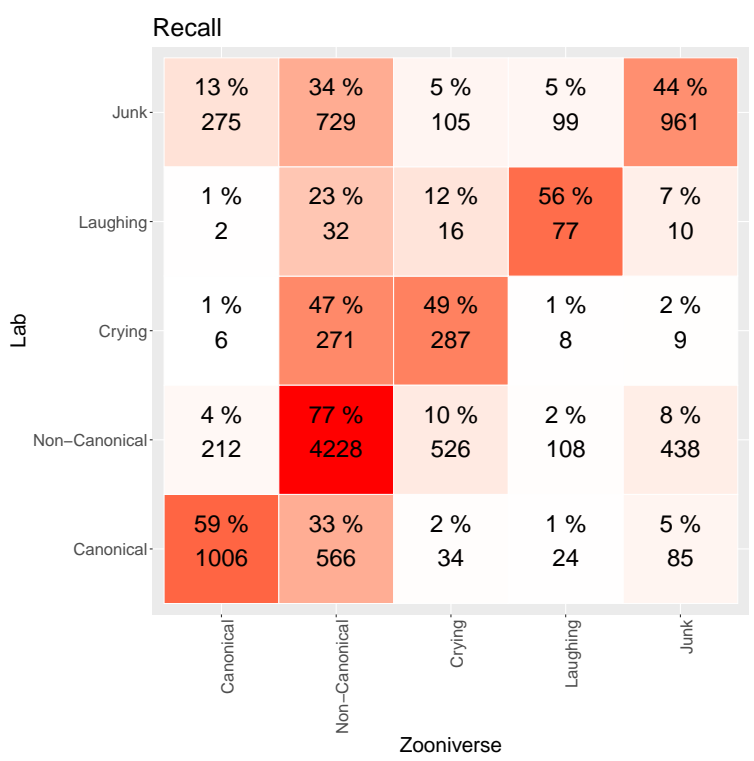

Fig. 2. Recall (i.e., category $X$ labels attributed by lab annotators that were classified as Y labels by Zooniverse annotators) in raw number of segments and percentage. in terms of Precision (Fig.1) and Recall (Fig.2): the diagonal elements show the number of correct classifications for each class and the off-diagonal elements show misclassifications. From both visualizations, it is evident that performance is moderate to good, with 65\% Accuracy (95\% CI .64-.66), a kappa of .4, and a No-Information Rate of 0.58. Errors seem to be systematic: the Non-Canonical class shows the highest agreement, and it is also the most common class in this dataset. The other classes have fewer segments assigned to them, but it appears that Junk and Canonical are quite well detected, with other classes having more variable assignments.

\subsection{Child-level descriptors}

Although there may be errors at the level of individual segments, what we really care about is whether Zooniverse annotations give a reliable image of the child's individual development. This is what we look at in this section. We look at two measures that have been found to relate to age and/or diagnosis: i) linguistic ratio = ("Canonical"+"NonCanonical")/“All vocalizations" (i.e., we remove junk), and ii) canonical ratio = "Canonical"/("Canonical"+"Non-Canonical") (i.e. we remove junk + non-linguistic vocalizations).

We found a strong positive correlation ( $\mathrm{r}=0.833$ ) between Linguistic ratio by child resulting from the Zooniverse and the lab annotators data, with errors suggesting that Zooniverse annotation may tend to under-estimate the linguistic ratio throughout the observed ratio range (see Fig. 3). Similarly, a strong positive correlation ( $\mathrm{r}=0.937)$ emerges from investigating the Canonical Ratio as well, and the error for Canonical Ratio is very small, with the regression line almost overlapping with the 45 degree line (see Fig. 4). These results show that Zooniverse annotations are able to capture the child individual variation as well as lab annotators.

\section{DISCUSSION}

In this study, we checked whether infant data collected from a wearable could be accurately annotated on Zooniverse, a platform enabling partnership with citizen scientists. We used a data set containing children varying in age and in whether they had a diagnosis, for whom excerpts from one day-long audiorecording had been annotated in the laboratory.

A first analysis found that, although classification of infants' individual vocalizations on Zooniverse was overall accurate as judged against the laboratory gold standard, some categories had relatively lower precision and/or recall. Nonetheless, an important advantage of Zooniverse compared to laboratory annotations is that it is easier to scale up, extracting more data from each individual child, to counter noise. Moreover, our second analysis suggested this may not even be necessary. Indeed, when we inspected descriptors that are defined at the level of individual children, we found very high correlations between descriptors derived 
$r=0.833$

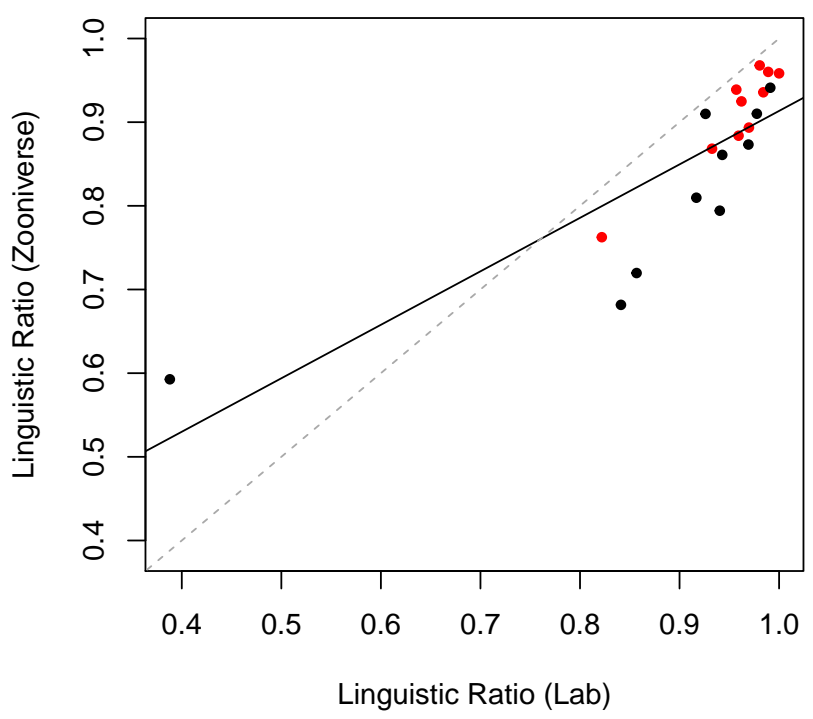

Fig. 3. Individual children's linguistic ratio according to Zooniverse versus Lab annotations. Black points correspond to children diagnosed with Angelman Syndrome, red for lowrisk control.

\section{$r=0.937$}

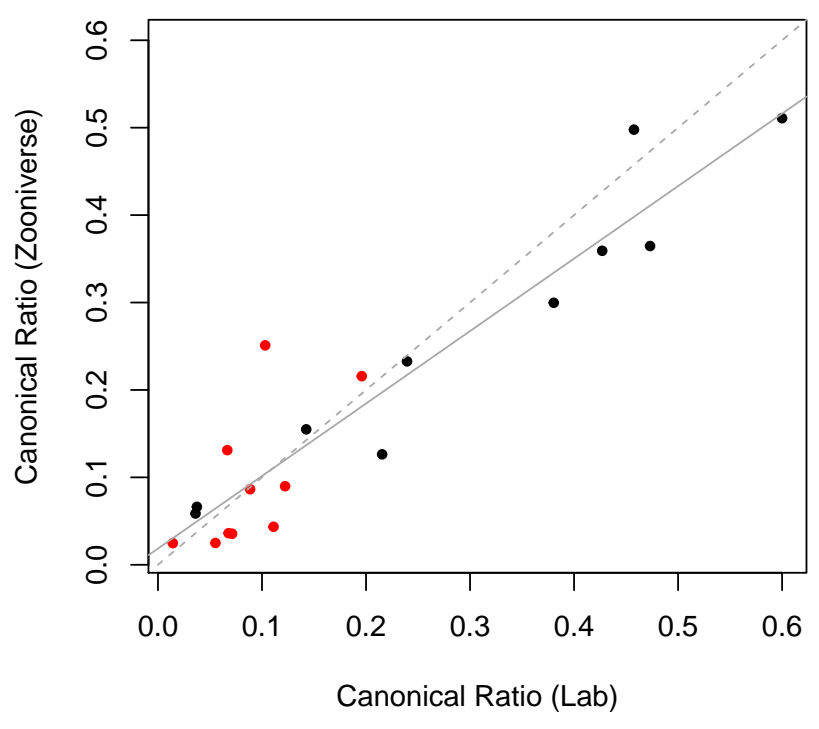

Fig. 4. Individual children's canonical ratio according to Zooniverse versus Lab annotations. Red points correspond to children diagnosed with Angelman Syndrome, black for low-risk control. from Zooniverse versus laboratory annotations, and relatively small errors. These results strongly support the continued use of Zooniverse for annotation of infant vocalizations, at least in terms of the descriptors studied here (linguistic and canonical ratio, which are most meaningful in the $0-5$ years age range).

Is the performance we observe comparable to current automated classifiers? Cychosz and colleagues collaborated with the ComParE team in the production of a BabySounds sub-task in the 2019 challenge [4], using the same five classes we employed here. The baseline performance was well below the weighted recall levels we observe here (see Table 1). We also inspected the accuracy of the LENA automated software (which yields a 4-way split, collapsing between canonical and non-canonical) on the same data used here, which obtained an intermediate level of performance. That said, the data produced by citizen scientists in this way can be used to train systems to automatically label even more data, perhaps by using Zooniverse annotations as weak labels to be integrated with stronger labels produced by laboratory annotations could significantly improve systems' performance at these challenging tasks [18].

Table 1. Performance in the ComParE BabySounds baseline, LENA software, and current annotations. Non-can stands for non-canonical. C2019BS stands for the ComParE baseline for the BabySounds 2019 subchallenge. LENA for LENA software annotations; *note LENA collapses across Canonical and Non-canonical. Zoon for the Zooniverse data in this paper. Lab indicates label frequency in laboratory annotations (on the data used here and for LENA evaluation). UAR stands for unweighted average recall and WAR for weighted average recall.

\begin{tabular}{l|ccc|cc} 
& \multicolumn{3}{|c|}{ Recall } & \multicolumn{2}{c}{ Label frequency } \\
& C2019B & LENA & Zoon. & C2019B & Lab \\
\hline Crying & 70.6 & 78.0 & 49.0 & 669 & 581 \\
Laughing & 41.5 & 12.0 & 56.0 & 149 & 137 \\
Non-can. & 24.1 & $73.0^{*}$ & 77.0 & 4485 & 5512 \\
Canonical & 66.4 & $*$ & 59.0 & 1426 & 1715 \\
Junk & 67.3 & 1.0 & 44.0 & 4575 & 2169 \\
\hline UAR & 54.0 & 41.0 & 57.0 & & \\
WAR & 49.9 & 57.0 & 65.0 & &
\end{tabular}

Given the promise of involving citizen scientists in classification tasks like those used here, it would be important to generalize this approach to investigations of other populations and other tasks. Regarding other populations, one may be interested in annotating potentially disordered speech by older adults at risk of neurodegenerative disorders. Although it would be important to similarly validate such annotations, we believe there is strong promise for such tasks, because many neurodegenerative disorders affect local aspects of the 
speech, which are detected at the syllable level. However, tasks that require more speech or audio context may not be well suited to Citizen Science platforms, because they would require playing longer clips, which may reveal identifying or sensitive information. Although citizen scientists are wellintentioned, the platform could be used by others who want to exploit the system for other means.

As for the possibility that these promising results generalize to other tasks, task complexity may be a crucial determinant. For instance, if we asked citizen scientists to transcribe children's speech or count syllables, this could have resulted in more errors for at least two reasons. First, such tasks would benefit from the whole vocalization being presented - which is not possible given ethical reasons as previously discussed. Second, they may also require greater training and attention from listeners, so that citizen scientists who just want to perform a couple of classifications may not come to learn how to do it well. This is something that can be investigated in follow-up work.

\section{CONCLUSION}

Current results suggest Zooniverse annotations of infant vocal sounds are fairly reliable, particularly when combined into child-level descriptors. This finding is particularly welcome in an era when wearables open new avenues for studying human behavior and development in an ecological manner.

\section{REFERENCES}

[1] Reza Rawassizadeh, Blaine A. Price, and Marian Petre, "Wearables: Has the age of smartwatches finally arrived?," Commun. ACM, vol. 58, no. 1, pp. 45-47, Dec. 2014.

[2] Dongxin Xu, Umit Yapanel, Sharmi Gray, Jill Gilkerson, Jeff Richards, and John Hansen, "Signal processing for young child speech language development," in First Workshop on Child, Computer and Interaction, 2008.

[3] Neville Ryant, Kenneth Church, Christopher Cieri, Alejandrina Cristia, Jun Du, Sriram Ganapathy, and Mark Liberman, "The second dihard diarization challenge: Dataset, task, and baselines," arXiv preprint arXiv:1906.07839, 2019.

[4] Björn W Schuller, Anton Batliner, Christian Bergler, Florian B Pokorny, Jarek Krajewski, Margaret Cychosz, Ralf Vollmann, Sonja-Dana Roelen, Sebastian Schnieder, Elika Bergelson, et al., "The interspeech 2019 computational paralinguistics challenge: Styrian dialects, continuous sleepiness, baby sounds \& orca activity.," in Interspeech, 2019, pp. 2378-2382.
[5] Elika Bergelson, Marisa Casillas, Melanie Soderstrom, Amanda Seidl, Anne S Warlaumont, and Andrei Amatuni, "What do north american babies hear? a largescale cross-corpus analysis," Developmental science, vol. 22, no. 1, pp. e12724, 2019.

[6] Jena McDaniel, Paul Yoder, Annette Estes, and Sally J Rogers, "Measuring early vocalizations (McDaniel et al., 2020)," 52020.

[7] Amanda Seidl, Anne Warlaumont, and Alejandrina Cristia, "Towards detection of canonical babbling by citizen scientists: Performance as a function of clip length," 09 2019, pp. 3579-3583.

[8] Adam J Berinsky, Gregory A Huber, and Gabriel S Lenz, "Evaluating online labor markets for experimental research: Amazon. com's mechanical turk," Political analysis, vol. 20, no. 3, pp. 351-368, 2012.

[9] Steven V Rouse, "A reliability analysis of mechanical turk data," Computers in Human Behavior, vol. 43, pp. 304-307, 2015.

[10] Yudhijit Bhattacharjee, "Citizen scientists supplement work of cornell researchers: a half-century of interaction with bird watchers has evolved into a robust and growing collaboration between volunteers and a leading ornithology lab," Science, vol. 308, no. 5727, pp. 14021404, 2005.

[11] Jonathan Silvertown, "A new dawn for citizen science," Trends in ecology \& evolution, vol. 24, no. 9, pp. 467471, 2009.

[12] Joe Cox, Eun Young Oh, Brooke Simmons, Chris Lintott, Karen Masters, Anita Greenhill, Gary Graham, and Kate Holmes, "Defining and measuring success in online citizen science: A case study of zooniverse projects," Computing in Science \& Engineering, vol. 17, no. 4, pp. 28-41, 2015.

[13] Anita Greenhill, Kate Holmes, Chris Lintott, Brooke Simmons, Karen Masters, Joe Cox, and Gary Graham, "Playing with science: Gamised aspects of gamification found on the online citizen science project-zooniverse," in GAMEON'2014. EUROSIS, 2014, pp. 15-24.

[14] Meg Cychosz, Alejandrina Cristia, Elika Bergelson, Marisa Casillas, Gladys Baudet, Anne S Warlaumont, Camila Scaff, Lisa Yankowitz, and Amanda Seidl, "Canonical babble development in a large-scale crosslinguistic corpus," 2019.

[15] DK Oller, "The emergence of the capacity for speech," 2000. 
[16] D Kimbrough Oller, Rebecca E Eilers, A Rebecca Neal, and Alan B Cobo-Lewis, "Late onset canonical babbling: A possible early marker of abnormal development," American Journal on Mental Retardation, vol. 103, no. 3, pp. 249-263, 1998.

[17] Dongxin Xu, Umit Yapanel, and Sharmi Gray, "Reliability of the lena language environment analysis system in young children's natural home environment," .

[18] Najla Al Futaisi, Zixing Zhang, Alejandrina Cristia, Anne Warlaumont, and Bjorn Schuller, "Vcmnet: Weakly supervised learning for automatic infant vocalisation maturity analysis," in 2019 International Conference on Multimodal Interaction, 2019, pp. 205-209. 